\title{
Analysis on Measures to Improve the Quality of Atmospheric Environment Monitoring
}

\section{Zhigang Huang}

Turpan Environmental Monitoring Station, Turpan, Xinjiang, 838000, China

\begin{abstract}
For today's environmental monitoring work, atmospheric environmental monitoring is one of the most important content. Because of its unique practicality, atmospheric environmental monitoring has brought great challenges to the current environmental monitoring staff. Researchers need to monitor different weather conditions and different air density, which are uncontrollable factors and will change at any time. Therefore, the actual quality achieved by the monitoring work becomes particularly important, so as to ensure the accuracy of the final relevant data.
\end{abstract}

\section{Keywords}

atmospheric environmental monitoring; monitoring quality; measures

\section{关于提高大气环境监测质量的措施分析}

\section{黄志刚}

吐鲁番市环境监测站，中国・新疆吐鲁番 838000

\section{摘 要}

对于现如今的环境监测工作来讲，大气环境监测是最主要的一项内容之一。大气环境监测工作由于其自身的独特实践性，给 当前开展环境监测的工作人员带来了极大挑战。科研人员需要就不同天气情况以及不同的空气密度情况开展监测工作, 这两 者本身都属于不可控因素, 也随时都会产生变化, 所以监测工作所取得的实际质量变得尤为重要, 从而保证最终所得相关数 据准确。

\section{关键词}

大气环境监测；监测质量；措施

\section{1 引言}

在自然环境属性当中, 空气以及水和生物都属于自然环 境的一份子, 对于人类来讲, 在对大自然进行改造的过程中, 主要目的是为了能够使生活及工作拥有更好的环境以及获得 更高的生活体验。现如今中国的自然环境条件, 人为的过度 开发正在不断恶化, 需要提高监测大气环境的治疗, 以此为 基础开展合理的大气环境治理工作。

\section{2 大气环境监测工作的内容}

20 世纪中后期, 很多国家逐渐建立起了环境自动监测系 统体系和生态监测系统体系, 利用环境自动监测技术准确发

【作者简介】黄志刚（1971-）, 中国广东惠州人, 本科学历, 工程师, 从事环境监测研究。
现了空气、水质以及生态环境的变化情况并及时进行了治理, 为人们工作生活环境质量甚至是全球生态环境质量做出了有 力的保障。通过分析大气污染物产生途径可知, 中国大比例 能源中, 石油和煤炭是主要来源, 但是中国社会的发展对此 类物质有着较大的需求量, 而中国发展中的重点产业之一为 农业, 汽车使用量不断增加, 所以每天都在源源不断地产生 二氧化硫和可吸入颗粒物, 在大气污染监测中, 重点监测内 容为二氧化硫、氮氧化物等。其中 PM 2.5 是人们熟知的颗粒 物监测内容, 可吸入颗粒物比二氧化硫有着更加复杂的组成 成分, 更加多样的形态, 如颗粒包括固体形态和液体形态, 监测过程中其可能会扩散到外界导致难以及时察觉。这些物 质在空气中的长期存留会严重危害到国民的身心健康, 同时 这些物质也会随着降雨产生酸雨现象而对其他的动植物生命 产生危害。石油和煤炭等燃烧是导致中国大气污染出现氮氧 
化物、二氧化硫超标的主要原因。此外, 氮氧化物等危害物 质另一来源为农业施肥和汽车尾气。可见在中国大气污染治 理工作中, 大气污染监测工作至关重要 ${ }^{[1]}$ 。

\section{3 大气环境污染存在的问题}

\section{1 资金问题}

对于现如今的环保部门来讲, 开展环境监测工作的过程 中, 是由财政部门负责拨款的, 但是对于财政部门来讲, 拨 款工作大多数都不积极, 而且拨款时款项的数量也不能够维 持或者是推动当前环保工作的进展，在当前环境监测工作当 中, 资金不足是制约大气环境监测, 不能够扩大监测工作规 模的最主要因素。

\section{2 生活污染}

在科学技术的持续发展背景下, 冰箱、空调等设备已经 成为中国人民日常生活中的重要部分, 但是在使用这些设备 的时候存在较严重的环境污染, 其运行过程产生大量的氟氯 烃气体, 而臭氧层受到这些气体的影响会出现被破坏的问题, 使得全球温室效应越加严重。同时, 在城市化进程快速发展 的情况下, 城市人口数量不断增多, 使得城市生活中的垃圾 数量与日俱增, 若无法及时对其进行处理, 势必会出现更多 的有害气体，使得大气环境受到破坏，难以保证中国人民的 身心健康。

\section{3 工业污染}

为保证中国在全球经济发展中的竞争力, 工业发展逐渐 加快, 但是在工业化发展的过程中, 普遍存在各种各样的环 境污染问题, 使得周边居民的生活质量受到较大影响。由于 环境保护管理制度不够完善, 且环保部门对环境污染的监管 力度不足, 部分企业未能严格按照要求对生产活动中的环境 污染问题进行处理, 使得硫化物等各种废气被直接排放到空 气中, 进一步加剧了大气环境污染的问题。同时, 由于生产 工艺的先进性、科学性及合理性不足, 导致工业生产活动中 的污染气体较多。基于这一情况, 需要保证工业生产废气排 放能够达到标准要求, 才能够缓解大气环境污染问题的不断 恶化。

\section{4 汽车尾气排放污染}

汽车是中国人民日常生活中比较常用的交通运输工具, 可以提高中国人民的出行效率，促进社会经济的快速发展。
但是在汽车的使用过程中, 汽车尾气的排放量对大气环境造 成了较大的破坏，很容易形成酸雨和温室气体等问题，最终 影响到中国人民的日常生活 ${ }^{[2]}$ 。一般在汽车使用的时候, 其 产生的尾气含有大量的硫化物、碳氢化合物等气体，这些气 体对人体存在较大的危害性, 且容易引起化学烟雾, 使得交 通能见度不断降低, 植物枯竭更加严重。

\section{4 大气环境监测质量提升对策}

\section{1 提高数据采集质量}

采样这一术语是环境监测工作当中的一种, 指针对空气 样本形成的集合。对于采样工作来讲可以分为多种不同的类 型, 一般在大气环境监测工作时, 数据采集的现场存在较大 的随机性, 导致数据采集面临着诸多不确定的因素, 其主要 体现在以下两个方面: 一方面是数据采集人员的专业素质不 足, 对数据采集的重视程度不高, 使得数据采集的质量难以 得到保障; 另一方面是数据采集中需要的设备数量较多, 在 资金有限的情况下存在设备更新不及时、设备维修不及时的 情况, 最终影响到设备获取数据的准确性, 给大气环境监测 数据的分析带来不利影响 ${ }^{[3]}$ 。

\section{2 严格控制采样时间及频率}

在当前的采样工作当中所应用的方法比较多, 无论是哪 种方法在实际应用的过程中都是需要落实到检测出空气质量 标准当中, 采样过程中的时间以及频率对最终的采样结果准 确性将会产生直接影响, 就是说若是没有将采样时间及频率 严格控制, 监测工作所得的污染物浓度就会出现偏差, 相应 的数据也会出现误差。以这些数据为基础所开展的分析工作, 就会出现较大的偏差, 不能够为环境改善工作提供参考 ${ }^{[4]}$ 。

\section{3 仪器设备的校准}

在大气环境监测工作当中所使用的相关仪器以及设备在 长期使用过程中，会使用频率过高而出现一定程度的误差， 如果没有及时针对设备进行校准以及调整, 在这种情况下所 取得的监测数据就可能会出现错误。为保证大气环境监测站 的建设水平, 需要适当增加对这一方面的资金投入, 使各个 地区都能够顺利做好环境监测, 提供可靠的数据信息。同时, 需要加强宣传工作, 强化工作人员的环保意识, 使其能够充 分认识到大气环境监测工作的重要意义, 为大气环境监测工 作的开展打下良好基础。此外, 需要加大环境监测技术的研 
究力度, 积极学习其他国家的新技术, 进一步促进中国大气 环境监测质量水平的提升。

\section{4 强化检测实验室质量控制及管理}

对于大气环境监测工作来讲, 实验室的主要用途是开展 监测及分析工作, 所以实验室的管理规格以及力度也需要全 面加强, 根据当前环境管理工作所提出的具体标准, 对实验 室管理工作进行完善, 增加实验室管理工作整体水平。对于 实验室内部来讲, 应当保证通风效果良好, 而且因为许多气 体当中含有有害成分, 所以需要在工作人员日常工作的过程 中, 对空气成分分析加强规范, 保证分析方法应用准确, 并 能够保障工作人员的安全。

\section{5 结语}

综上所述, 对于大气环境监测工作来讲, 虽然工作复杂
程度比较高, 比较辛苦, 最主要的目的都是为了能够使中国 当前的环境质量得到改善, 为经济发展打下坚实的基础, 大 气环境监测工作需要认真对待, 对每一个工作细节给予充分 的重视并发现问题，及时改正，使大气环境监测工作水平得 到全面提升。

\section{参考文献}

[1] 黄胜银. 提高大气环境监测质量的措施分析 [J]. 建材与装 饰 , 2016,49(105):151-152.

[2] 徐琴容. 提高大气环境监测质量的措施探讨 [J]. 工业, 2016(06):78.

[3] 冯翌铎. 浅析提高大气环境监测质量的主要措施 [J]. 民营科 技, 2016(02):229.

[4] 黄海波. 提高大气环境监测质量的措施分析 [J]. 化工设计通 讯 ,2017(08):200+232. 